\title{
Reproduction of Turbinate Atrophy in Pigs by Intranasal Consecutive-Day Inoculation with Suspensions of a Toxigenic Serotype D Strain of Pasteurella multocida
}

Toru KAWAI, Takashi HONDA, Yukio TOKUYAMA, Akira TANENO, Sinji OISHI, Hiroshi OKAMURA, Fujio NONAKA, Eishi SAKAI, Tokuji MIYAHARA, and Masanobu ETO

Department of Animal Products, The Chemo-Sero-Therapeutic Research Institute, 668 Okubo, Shimizu-machi, Kumamoto 860, Japan (Received 24 April 1990/Accepted 5 February 1991)

KEY WORDS: atrophic rhinitis, swine nasal turbinate, toxigenic Pasteurella multocida.

Porcine atrophic rhinitis (AR) is a disease characterized by hypoplasia or disappearance of the turbinate bones. Bordetella bronchiseptica has been identified as a primary causative agent of the disease. The dermonecrotic toxin (DNT) of B. bronchiseptica was considered as a major pathogenic factor responsible for the production of nasal turbinate atrophy [8]. de Jong et al. [5] reported that some strains of Pasteurella multocida produced DNT and caused severe atrophy of the nasal turbinates of pigs. The nasal lesion was induced by intranasal or intramuscular injection with the purified DNT from the organism $[6,9]$. The importance of toxigenic $P$. multocida in AR has been accepted by researchers in many countries [4]. However, it was difficult to reproduce the turbinate atrophy by the experimental infections with toxigenic $P$. multocida, because the organism had a poor affinity to swine nasal mucosa and epithelial cells [7]. For colonization of the organism, combined infection with toxigenic $P$. multocida and $B$. bronchiseptica [1], damage with a nasal irritant such as diluted acetic acid [13] and consecutive-day inoculation $[2,3,9,12,16]$ were required. It was thought that AR was transmitted by way of infective aerosols and that the severity of the clinical expression of AR was dependent on the frequency of the aerosol transmission of AR pathogens in the swine herd [3]. Therefore, the consecutive-day inoculation method was chosen in this study. By this method, older broth cultures of toxigenic $P$. multocida were generally used as inocula $[2,3,9,12]$. Even with broth-culture filtrates, the turbinate atrophy was induced in piglets $[2,3,12]$. However, it was difficult to induce the nasal lesion by the inocula consisting of suspensions of the organism grown on agar medium [12]. These findings suggest that DNT released from the organism into broth culture medium may give some influences on the turbinate bones. Thus, the purpose of the present study is to determine the conditions of induction of turbinate atrophy in SPF pigs by intranasal consecutive-day inoculation with suspensions of a toxigenic strain of $P$. multocida capsular serotype D grown on agar medium.

Toxigenic $P$. multocida strain 70 of capsular serotype D, somatic serotype 11 , which was isolated from a porcine pneumonic lesion at a slaughterhouse in Nagasaki Prefecture in 1983 was kindly supplied by Dr. S. Iwamatsu (Chuo Livestock Hygiene Service Center of Nagasaki Prefecture, Japan). This strain had no fimbriae or hemag- glutinating property with human type $\mathrm{O}$ red blood cells. The DNT sonically extracted from strain 70 (concentration of $10^{10}$ cells per $\mathrm{m} l$ ) had a titer of 32 in $0.1 \mathrm{~m} l$ by the guinea pig skin test [5]. The organism propagated on dextrose starch agar (Difco, Detroit, U.S.A.) plates at $37^{\circ} \mathrm{C}$ for $6 \mathrm{hr}$ was harvested, suspended in heart infusion broth (Difco) and used for inoculum.

In experiment 1 , eleven 1-day-old gnotobiotic piglets produced by hysterectomy were divided into 3 groups. The 1 st and 2 nd groups were inoculated intranasally with $10^{7}$ viable organisms of strain $70(0.5 \mathrm{~m} / /$ each nostril $)$ during 3 and 6 consecutive days, respectively. The 3rd group was uninoculated control.

The piglets were reared in sterile stainless steel isolators until the age of 3 weeks and thereafter kept in individual rooms. The piglets were sacrificed at the age of 14,21 or 35 days.

In experiment 2, ten 1-day-old SPF piglets were produced by immediate removal from sows at the birth and raised in individual rooms. Seven piglets were inoculated intranasally with $10^{4}, 10^{6}, 10^{8}$ or $10^{10}$ viable organisms of strain $70(0.5 \mathrm{~m} / /$ each nostril $)$ during 6 consecutive days. The remaining three piglets were used as uninoculated controls. All the piglets were sacrificed at the age of 45 days.

In experiment 3 , seven 80 -day-old SPF pigs obtained from our breeding herd were raised in individual rooms. Six pigs were inoculated intranasally with $2 \times 10^{7}, 2 \times 10^{9}$ or $2 \times 10^{10}$ viable organisms of strain $70(1 \mathrm{ml} / \mathrm{each}$ nostril $)$ during 6 consecutive days. The remaining one pig was used as an uninoculated control. All the pigs were sacrificed at the age of 122 days.

The snouts of the sacrificed pigs were sectioned transversely at the site of the first upper premolar teeth. Macroscopic findings of atrophy of nasal turbinates were graded according to the criteria described by Maeda $e t$ al. [10]. The recovery of strain 70 from the nasal cavities was performed with sterile cotton-tipped swabs at necropsy.

In experiment 1 , the 2 nd group inoculated with strain 70 during 6 consecutive days had a very severe turbinate atrophy, although the 1st group during 3 consecutive days had moderate gross lesions (Table 1). The organism was recovered from 2 of 4 piglets in small numbers (about $10^{4}$ $\mathrm{cfu} / \mathrm{g}$ of nasal exudate) in the 1st group and all of 5 piglets in large numbers $\left(10^{6}-10^{8} \mathrm{cfu} / \mathrm{g}\right)$ in the 2 nd group. The $3 \mathrm{rd}$ group pigs as uninoculated controls had no gross nasal lesion and were bacteriologically negative for the organism. None of these groups piglets showed any clinical signs of AR during the experimental period. 
Table 1. Results of intranasal inoculation with strain 70 of $P$. multocida serotype D in pigs

\begin{tabular}{|c|c|c|c|c|c|}
\hline $\begin{array}{c}\text { Experiment } \\
\text { No. }\end{array}$ & $\begin{array}{c}\text { Dose } \\
\text { of } \\
\text { inoculum }\end{array}$ & $\begin{array}{l}\text { Age in } \\
\text { days for } \\
\text { inoculation }\end{array}$ & $\begin{array}{c}\text { Age in } \\
\text { days at } \\
\text { necropsy }\end{array}$ & $\begin{array}{l}\text { Recovery of } \\
\text { strain } 70 \\
\text { from nasal cavity }\end{array}$ & $\begin{array}{c}\text { Grade of } \\
\text { atrophy of } \\
\text { nasal turbinates }\end{array}$ \\
\hline \multirow[t]{6}{*}{1} & $10^{7 a)}$ & $1-3^{b)}$ & 14 & $1 / 2^{\mathrm{c})}$ & $H, H^{d)}$ \\
\hline & $10^{7}$ & $1-3$ & 35 & $1 / 2$ & H, H \\
\hline & $10^{7}$ & $1-6$ & 14 & $1 / 1$ & HI \\
\hline & $10^{7}$ & $1-6$ & 21 & $3 / 3$ & m, , mt, $\mathrm{m}$ \\
\hline & $10^{7}$ & $1-6$ & 35 & $1 / 1$ & mt \\
\hline & - & - & 35 & $0 / 2$ &,-- \\
\hline \multirow{5}{*}{2} & $10^{4}$ & $1-6$ & 45 & $0 / 2$ &,-- \\
\hline & $10^{6}$ & $1-6$ & 45 & $0 / 2$ & mI, $\mathrm{m}$ \\
\hline & $10^{8}$ & $1-6$ & 45 & $0 / 2$ & m, , H \\
\hline & $10^{10}$ & $1-6$ & 45 & $0 / 1$ & m \\
\hline & - & - & 45 & $0 / 3$ &,,--- \\
\hline \multirow[t]{4}{*}{3} & $2 \times 10^{7}$ & $80-85$ & 122 & $0 / 2$ &,-- \\
\hline & $2 \times 10^{9}$ & $80-85$ & 122 & $0 / 2$ &,-- \\
\hline & $2 \times 10^{10}$ & $80-85$ & 122 & $1 / 2$ &,++ \\
\hline & - & - & 122 & $0 / 1$ & - \\
\hline
\end{tabular}

a) Cells/dose. The bacterial concentrations were spectrophotometrically adjusted.

b) Inoculated daily during the ages.

c) No. of pigs which recovered the inoculated organism/No. tested.

d) Macroscopically graded according to the criteria described by Maeda et al. ( $-=$ normal, $+=$ mild, $H=$ moderate, $H=$ severe, $m=$ very severe) [10].

In experiment 2 , the piglets inoculated with $10^{4}$ viable organisms of strain 70 and uninoculated controls had no nasal lesions. However, the piglets inoculated with $10^{6}$ to $10^{10}$ viable cells had severe or very severe turbinate atrophy (Table 1). The degree of nasal lesions induced by inoculations with $10^{6}$ or more viable organisms was not dependent on the concentrations of viable organisms in the inocula. In spite of such severe nasal lesions, none of the piglets showed any clinical manifestations of AR. No inoculated organism was recovered from the nasal cavities of any piglets.

In experiment 3 , the pigs inoculated with $2 \times 10^{7}$ or $2 \times 10^{9}$ viable organisms of strain 70 and an uninoculated control had no nasal lesions. Two pigs inoculated with $2 \times 10^{10}$ viable organisms had a mild turbinate atrophy, and the inoculated organism was recovered from only one of the pigs (Table 1). No clinical signs were observed in these pigs.

Several reports showed that induction of nasal turbinate atrophy in pigs by broth cultures of toxigenic $P$. multocida was easier than that by the suspensions of viable organisms of toxigenic $P$. multocida grown on agar medium [11, 12]. In the present study, however, it was demonstrated that 1-day-old SPF piglets induced the nasal lesion by intranasal consecutive-day inoculation of the bacterial suspensions. The degree of the nasal lesions was dependent on the repeated inoculation times and the age of piglets used (Table 1). In relation to the age, de Jong and Akkermans [3] had already reported. The inoculated organism was recovered from only 3 of 16 pigs (18.8\%) kept in individual rooms, though done from 5 of 6 pigs $(83.3 \%)$ kept in isolators. It was indicated that some normal microbial flora eliminated $P$. multocida from the nasal mucosa and that the colonization by the consecutive- day inoculation was as temporary as that by the inoculation following intranasal treatment with diluted acetic acids [15]. The temporality of colonization of $P$. multocida may be due to the poor affinity to swine nasal epithelial cells [7]. However, turbinate atrophy could be caused by the maintenance of sufficiently large numbers of toxigenic $P$. multocida in nasal cavity for a few days [1].

In Japan, there has been no report on the successful induction of turbinate atrophy in pigs using toxigenic $P$. multosida alone. Etiologically, no correlation between appearance of AR in pigs and DNT-producing ability of $P$. multocida was observed [16]. Thus, some Japanese researchers have questioned the role of the bacterium in AR [13, 14, 16]. Oyamada et al. [14] reported that no gross lesions of the nasal turbinate bones were observed in all the pigs inoculated with the bacterial suspensions of 5 $\times 10^{7}$ and $1.5 \times 10^{8}$ viable organisms 1 and 3 times at weekly intervals, respectively, although the organism was constantly recovered in low numbers from the nasal cavities of the pigs during the experimental period. This may be due to the low grade of colonization. Nakagawa et al. [13] found no significant lesions in gnotobiotic piglets inoculated with serotype A strain Kobe 5 of $P$. multocida. The reason of their failure may be related to the inability of the strain used to produce DNT (data not shown).

The inoculation method demonstrated in the present study may be applicable to estimation of the efficacy of a toxigenic $P$. multocida vaccine maternally used.

ACKNOWLEDGEMENTS. The authors wish to thank Dr. Y. Tokiyoshi for reviewing the manuscript. 


\section{REFERENCES}

1. Chanter, N. and Rutter, J. M. 1989. pp. 161-195. In: Pasteurella and Pasteurellosis (Adlam, C. and Rutter, J. M. eds.), Academic Press, London.

2. de Jong, M. F. 1983. pp. 136-146. In: Atrophic Rhinitis in Pigs (Pedersen, K. B. and Nielsen, N. C. eds.), Commission of European Communities, Brussels, Belgium.

3. de Jong, M. F. and Akkermans, J. P. W. M. 1986. Vet. Quart. 8: 204-214.

4. de Jong, M. F. and Nielsen, J. P. 1990. Vet. Rec. 126: 93.

5. de Jong, M. F., Oei, H. L., and Tetenburg, G. J. 1980. Proc. 5th Int. Pig Vet. Soc. (Copenhagen, Denmark) 211.

6. Dominik, M. A. and Rimler, R. B. 1986. Am. J. Vet. Res. 47: 1532-1536.

7. Frymus, T., Wittenbrink, M-M., and Petzoldt, K. 1986. $J$. Vet. Med. B 33: 140-144.

8. Hanada, M., Shimoda, S., Tomota, S., Nakase, Y., and Nishiyama, Y. 1979. Jpn. J. Vet. Sci. 41: 1-8.
9. Kamp, E. M. and Kimman, T. G. 1988. Am. J. Vet. Res. 49: 1844-1849.

10. Maeda, M., Inui, S., and Konno, S. 1969. Natl. Inst. Anim. Health Q. (Jpn.) 9: 193-202.

11. Martineau, G. P., Broes, A., de Jong, M. F., and Martineau-Doize, B. 1982. Proc. 6th Int. Pig Vet. Soc. (Mexico) 88 .

12. Martineau, G. P., Martineau-Doize, B., and Broes, A. 1985. Zentralbl. Veterinaermed., B 32: 583-592.

13. Nakagawa, M., Shimizu, T., and Motoi, Y. 1974. Natl. Inst. Anim. Health $Q$. (Jpn.) 14: 61-71.

14. Oyamada, T., Yoshikawa, T., Yoshikawa, H., Shimizu, M., Nakai, T., and Kume, K. 1986. Jpn. J. Vet. Sci. 48: 377-387.

15. Pedersen, K. B. and Elling, F. 1984. J. Comp. Pathol. 94: 203-214.

16. Sawata, A., Nakai, T., Tsuji, M., and Kume, K. 1984. Jpn. J. Vet. Sci. 46: 141-148. 